\title{
RATIONAL FIBRATIONS, MINIMAL MODELS, AND FIBRINGS OF HOMOGENEOUS SPACES ${ }^{1}$
}

BY

\author{
STEPHEN HALPERIN
}

\begin{abstract}
Sullivan's theory of minimal models is used to study a class of maps called rational fibrations, which contains most Serre fibrations.

It is shown that if the total space has finite rank and the fibre has finite dimensional cohomology, then both fibre and base have finite rank.

This is applied to prove that certain homogeneous spaces cannot be the total space of locally trivial bundles.

In addition two main theorems are proved which exhibit a close relation between the connecting homomorphism of the long exact homotopy sequence, and certain properties of the cohomology of fibre and base.
\end{abstract}

1. Introduction. Originally Sullivan's theory of minimal models [12] was a technique for the study of topological spaces. First he defined a contravariant functor $S \leadsto(A(S), d)$ from spaces to commutative graded differential algebras (c.g.d.a.'s) over a fixed field $k$ of characteristic zero.

Then with each c.g.d.a. $\left(G, d_{G}\right)$ such that $H^{0}(G)=k$ he associated a homomorphism

$$
\varphi:(\Lambda X, d) \rightarrow\left(G, d_{G}\right)
$$

such that $\varphi^{*}$ is an isomorphism, $\Lambda X$ is the free commutative graded algebra (c.g.a.) over $X, \operatorname{Im} d \subset \Lambda^{+} X \cdot \Lambda^{+} X$ and a certain "nilpotence" condition is satisfied (cf. (2.3) for a precise definition). This determines $(\Lambda X, d)$ up to isomorphism, and (1.1) is called the minimal model for $\left(G, d_{G}\right)$. (Complete definitions are in $\$ 2$ below.)

If $S$ is a space $H^{*}(S ; k)$ always denotes its singular cohomology (coefficients in $k$ ). If $S$ is path connected the minimal model $(\Lambda X, d)$ of $(A(S), d)$ satisfies $H(\Lambda X) \cong H(A(S)) \cong H^{*}(S ; k)$. If $S$ is 1-connected and $H^{*}(S ; k)$ has finite type then also

$$
X \simeq \operatorname{Hom}_{z}\left(\pi_{*}(S) ; k\right) \text {. }
$$

Now in [12] Sullivan generalizes this to the minimal model of a homomorphism $\gamma:\left(G, d_{G}\right) \rightarrow\left(L, d_{L}\right)$ between c.g.d.a.'s; this is a commutative

Received by the editors February 28, 1977.

AMS (MOS) subject classifications (1970). Primary 55F20, 55J99, 57F15.

Key words and phrases. Rational fibration, minimal model, connecting homomorphism, spaces of finite rank, homogeneous space.

'Supported by NRC grant no. A8047. 
diagram of c.g.d.a. homomorphisms

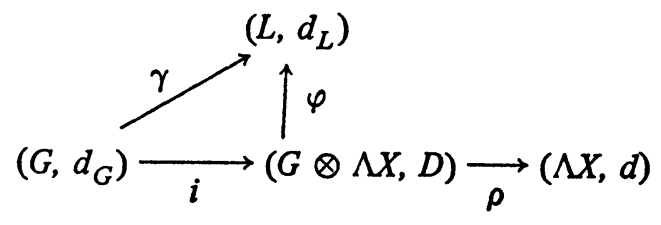

in which $\varphi^{*}$ is an isomorphism. It is the exact analogue of the technique of converting a continuous map into a fibration.

If $F \rightarrow^{j} E \rightarrow \pi^{\pi} B$ is a sequence of continuous maps such that $\pi \circ j$ is the constant map then we apply the above procedure to $A(\pi)$ and obtain a commutative diagram

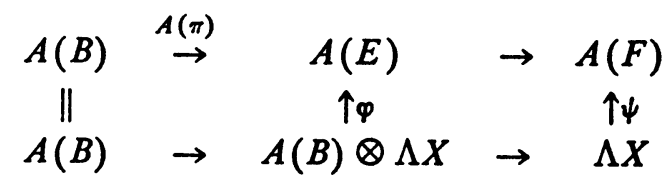

in which $\varphi^{*}$ is an isomorphism but $\psi^{*}$ need not be. If $\psi^{*}$ is an isomorphism we say $F \rightarrow^{j} E \rightarrow^{\pi} B$ is a rational fibration. (For a technically complete definition see Definition 4.5 below.)

A theorem of Grivel [5] asserts that a Serre fibration of path connected spaces and 1-connected base with either base or fibre having rational cohomology of finite type is a rational fibration. This is generalized to non-simply-connected bases in [8] (see Theorem 4.6 below).

The purpose of this paper is to analyse rational fibrations. Our main technique (cf. §3) is the $\Lambda$-model, in which we replace $A(B)$ by its minimal model in (1.3) to obtain a sequence

$$
\Lambda Y \rightarrow \Lambda Y \otimes \Lambda X \rightarrow \Lambda X
$$

of c.g.d.a.'s. This gives a convenient interpretation of the dualized rationalized long exact homotopy sequence-cf. (4.9) below.

In particular we obtain as special cases of our main theorems (Theorems 4.15 and 4.17 below) the following:

1.4. TheOREM. Let $F \rightarrow \rightarrow^{\prime} E \rightarrow{ }^{\pi} B$ be a Serre fibration, and let $\partial: \pi_{k}(B) \rightarrow$ $\pi_{k-1}(F)$ be the connecting homomorphism. Assume $F, E, B$ are 1-connected $C W$ complexes and that $H^{*}(F ; \mathbf{Q}), H^{*}(E ; \mathbf{Q})$ and $H^{*}(B ; \mathbf{Q})$ are graded spaces of finite type. Then:

(i) If Coker $\partial$ is strictly torsion then there is an isomorphism of graded algebras

$$
H^{*}(F ; \mathbf{Q}) \approx \Lambda\left(\pi_{*}(F) \otimes \mathbf{Q}\right) .
$$

(ii) If $\operatorname{Im} \partial$ is strictly torsion and $H^{p}(E ; \mathrm{Q})=0, q>N$, then any $N+1$ 
cohomology classes, $\gamma_{1}, \ldots, \gamma_{N+1}$, in $H^{+}(F ; \mathbf{Q})$ have product zero: $\gamma_{1} \cdot \cdots \cdot \gamma_{N+1}=0$.

(iii) If $\operatorname{dim} H^{*}(F ; \mathbf{Q})<\infty$ then $\partial\left(\pi_{\text {odd }}(B)\right)$ is strictly torsion.

1.5. THEOREM. Let $\varphi: S \rightarrow T$ be a continuous map between 1-connected $C W$ complexes such that $H^{*}(S ; \mathbf{Q})$ and $H^{*}(T ; \mathbf{Q})$ are graded spaces of finite type. Assume $H^{p}(T ; Q)=0, p>N$, and $\varphi_{\#}: \pi_{*}(S) \rightarrow \pi_{*}(T)$ has kernel which is strictly torsion.

Then the product of any $N+1$ cohomology classes $\gamma_{1}, \ldots, \gamma_{N+1}$ in $\mathrm{H}^{+}(S$; $k$ ) is zero: $\gamma_{1} \cdot \cdots \cdot \gamma_{N+1}=0$.

A space $S$ is said to have finite rank if its minimal model is generated by a finite dimensional space $X$. Homogeneous spaces $G / K(G$ and $K$ connected Lie groups) are examples. We obtain

4.15(iv). THEOREM. Suppose $F \rightarrow E \rightarrow B$ is a rational fibration in which $E$ has finite rank and $H^{p}(F ; k)=0, p>N$. Then $F$ and $B$ have finite rank.

This places a severe restriction on the possible fibrings of a homogeneous space. Indeed we establish as an application

7.7. THEOREM. The following manifolds do not appear as the total space of a locally trivial bundle (with connected fibre) over a CW complex unless either fibre or base is a single point:

(i) $S^{2 k}, k>1$. (ii) $\mathrm{C}^{p-1}, p$ a prime. (iii) $U(4) / U(2) \times U(2)$.

The paper is organized as follows. In $\$ 2$ the basic facts from Sullivan's theory are recalled. Throughout [8] is cited as a reference, solely because it contains detailed proofs: the theorems are due to Sullivan [12].

In $\$ 3$ we develop the notion of $\Lambda$-model, and in $\$ 4$ we define rational fibration and state the main theorems. For technical reasons we work always in the categories of pointed spaces and augmented c.g.d.a.'s.

$\$ 5$ contains the proofs of the results of $\$ 4$. In $\$ 6$ we apply the theory to spaces of finite rank and in $\$ 7$ we specialize to homogeneous spaces.

Theorems 1.4 and 1.5 may be regarded as showing how the connecting homomorphism (in homotopy) of a fibration influences the cohomology of the fibre and total space (over Q). It is interesting to wonder if there are analogous results in characteristic different from zero.

It would also be interesting to have a complete list of compact homogeneous spaces which do not appear as the total space of any locally trivial bundle.

2. Minimal models. We always work over a fixed field $k$ of characteristic zero. Graded spaces are graded over the nonnegative integers, which are 
written as superscripts. All differentials will have degree +1 , and all algebras are associative with identity.

A commutative graded algebra satisfies $a b=(-1)^{\operatorname{deg} a \operatorname{deg} b} b a$, and tensor product means in the graded commutative category. We use c.g.d.a. for commutative graded differential algebra $\left(A, d_{A}\right)$.

A homomorphism $\varphi:\left(A, d_{A}\right) \rightarrow\left(A^{\prime}, d_{A^{\prime}}\right)$ induces a homomorphism $\varphi^{*}$ : $H(A) \rightarrow H\left(A^{\prime}\right)$.

If $X$ is a graded space $\Lambda X$ denotes the free c.g.a. over $X: \Lambda X=$ exterior algebra $\left(X^{\text {odd }}\right) \otimes$ symmetric algebra $\left(X^{\text {even }}\right)$.

If $A$ is a c.g.a. augmented by $\varepsilon_{A}$ we set

$$
Q(A)=\operatorname{ker} \varepsilon_{A} / \operatorname{ker} \varepsilon_{A} \cdot \operatorname{ker} \varepsilon_{A}
$$

and denote by $\zeta_{A}: \operatorname{ker} \varepsilon_{A} \rightarrow Q(A)$ the projection. If $A$ is a c.g.d.a. then $d_{A}$ induces a differential $Q\left(d_{A}\right)$ in $Q(A)$, and $\zeta_{A}$ gives a linear map

$$
\zeta_{A}^{*}: H\left(\operatorname{ker} \varepsilon_{A}\right) \rightarrow H(Q(A))
$$

A $K S$ extension is a sequence

$$
\mathcal{E}:\left(G, d_{G}, \varepsilon_{G}\right) \stackrel{i}{\rightarrow}\left(C, d_{C}, \varepsilon_{C}\right) \stackrel{\rho}{\rightarrow}\left(A, d_{A}, \varepsilon_{A}\right)
$$

of homomorphisms of augmented c.g.d.a.'s which satisfy the following:

(2.1) For some graded space $X \subset \operatorname{ker} \varepsilon_{A}, A=\Lambda X$.

(2.2) There is an isomorphism of graded augmented algebras, $f: C \simeq G \otimes$ $\Lambda X$, compatible with $i$ and $\rho$.

(2.3) There is a well-ordered homogeneous basis $\left\{x_{\alpha}\right\}_{\alpha \in \mathcal{G}}$ of $X$ such that (with respect to $f$ )

$$
d_{C}\left(1 \otimes x_{\alpha}\right) \in G \otimes(\Lambda X)_{<\alpha}, \quad \alpha \in \mathcal{G} .
$$

(Note. $(\Lambda X)_{<\alpha}$ is the subalgebra generated by the $x_{\beta}, \beta<\alpha$. Similarly we have $\left.(\Lambda X)_{<\alpha},(\Lambda X)_{>\alpha},(\Lambda X)_{>\alpha}\right)$

The augmented c.g.d.a.'s $G, C$ and $A$ are called respectively the base, total and fibre algebras of $\mathcal{E}$.

A $K S$ extension $\mathcal{E}$ is called minimal if the basis in (2.3) can be chosen so that $\operatorname{deg} x_{\beta}<\operatorname{deg} x_{\alpha}$ implies $\beta<\alpha$. If $H^{0}(G)=H^{0}(C)=k$ this is equivalent to $Q\left(d_{A}\right)=0$ (cf. [8, Corollary 2.4D).

If $G=k$ (so that $C=A=\Lambda X$ ) we say $\left(C, d_{C}, \varepsilon_{C}\right)$ is a $K S$ complex (resp. a minimal $K S$ complex). In particular the fibre of a $K S$ extension $\mathcal{E}$ is a $K S$ complex, minimal if $\mathcal{E}$ is minimal.

Now suppose

$$
\gamma:\left(G, d_{G}, \varepsilon_{G}\right) \rightarrow\left(L, d_{L}, \varepsilon_{L}\right)
$$

is a homomorphism of augmented c.g.d.a.'s, and assume that $H^{0}(G)=$ $H^{0}(L)=k$. A model for $\gamma$ is a $K S$ extension 


$$
\mathcal{E}: G \stackrel{i}{\rightarrow} C \stackrel{\rho}{\rightarrow} A,
$$

together with a homomorphism of augmented c.g.d.a.'s

$$
\varphi:\left(C, d_{C}, \varepsilon_{C}\right) \rightarrow\left(L, d_{L}, \varepsilon_{L}\right)
$$

such that $\varphi \circ i=\gamma$ and $\varphi^{*}: H(C) \rightarrow H(L)$ is an isomorphism.

If $\mathcal{E}$ is minimal we say $(\mathscr{E}, \varphi)$ is the minimal model for $\gamma$. The homomorphism $\gamma$ always has a minimal model, and its minimal model is unique up to isomorphism (cf. [8, Theorems 6.1, 6.2]).

If $\gamma$ is the inclusion of $k$ in $L$ then a model is just a homomorphism $\varphi$ : $\left(C, d_{C}, \varepsilon_{C}\right) \rightarrow\left(L, d_{L}, \varepsilon_{L}\right)$ where $C$ is a $K S$ complex and $\varphi^{*}$ is an isomorphism. We say $(C, \varphi)$ is a model (resp. minimal) for $\left(L, d_{L}, \varepsilon_{L}\right)$.

Suppose $H^{0}(L)=k$ and that $\left(C_{j}, \varphi_{j}\right)$ are models for $\left(L, d_{L}, \varepsilon_{L}\right)$. Then by $[8$, Theorem 5.20] there is a homomorphism $\varphi: C_{1} \rightarrow C_{2}$ of augmented c.g.d.a.'s such that $\varphi_{2} \varphi$ is based homotopic to $\varphi_{1}$ (cf. [8, Chapter 5] for the definition). In particular, this implies that $\varphi^{*}: H\left(C_{1}\right) \rightarrow H\left(C_{2}\right)$ and $Q(\varphi)^{*}: H\left(Q\left(C_{1}\right)\right) \rightarrow$ $H\left(Q\left(C_{2}\right)\right)$ are isomorphisms. Moreover (cf. [8, Lemma 8.3]), the isomorphism $Q(\varphi)^{*}$ is independent of the choice of $\varphi$.

Thus if we write $\pi_{\psi}^{*}(L ; C, \varphi)=H^{*}(Q(C))$ we have a canonical identification $\pi_{\psi}^{*}\left(L ; C_{1}, \varphi_{1}\right) \cong \pi_{\psi}^{*}\left(L ; C_{2}, \varphi_{2}\right)$. Thus we may identify all these spaces as a single graded space, which we denote by $\pi_{\psi}^{*}(L)$. If $(C, \varphi)$ is the minimal model then $\pi_{\psi}^{*}(L)=Q(C)$.

2.4. Definition. $\pi_{\psi}^{*}(L)$ is called the $\psi$-homotopy space of the augmented c.g.d.a. $L$.

Now assume $\eta:\left(G, d_{G}, \varepsilon_{G}\right) \rightarrow\left(L, d_{L}, \varepsilon_{L}\right)$ is a homomorphism of augmented c.g.d.a.'s, with $H^{0}(G)=k=H^{0}(L)$. If $\left(C_{1}, \varphi_{1}\right)$ is a model for $G$ and $\left(C_{2}, \varphi_{2}\right)$ is a model for $L$ then there is a homomorphism of augmented c.g.d.a.'s $\varphi$ : $C_{1} \rightarrow C_{2}$ such that $\varphi_{2} \varphi$ is based homotopic to $\eta \varphi_{1}$ (cf. [8, Theorem 5.20]). By [8, Lemma 8.3], $Q(\varphi)^{*}$ is independent of the choice of $\varphi$.

The linear maps $Q(\varphi)^{*}: \pi_{\psi}^{*}\left(G ; C_{1}, \varphi_{1}\right) \rightarrow \pi_{\psi}^{*}\left(L ; C_{2}, \varphi_{2}\right)$ are compatible with the identifications defined above [8, Chapter 8$]$ and so define a unique linear map

$$
\eta^{\#}: \pi_{\psi}^{*}(G) \rightarrow \pi_{\psi}^{*}(L) \text {. }
$$

Moreover $\eta_{1}^{\#} \eta_{2}^{\#}=\left(\eta_{1} \eta_{2}\right)^{\#}$ and $\iota^{\#}=\iota$; thus $\psi$-homotopy is a functor from augmented c.g.d.a.'s with connected cohomology to strictly positively graded spaces.

3. $\Lambda$-models. A $\Lambda$-extension is a $K S$ extension in which the base is itself a $K S$ complex; it is called $\Lambda$-minimal if both the base and the extension are minimal.

A $\Lambda$-extension $\mathcal{E}: G \rightarrow^{i} C \rightarrow^{\rho} A$ can be written in the form

$$
Y \stackrel{i}{\rightarrow} \Lambda Y \otimes \Lambda X \stackrel{\rho}{\rightarrow} \Lambda X,
$$


where $Y$ and $X$ admit well-ordered homogeneous bases $\left\{y_{\gamma}\right\}_{\gamma \in \mathcal{G}}$ and $\left\{x_{\alpha}\right\}_{\alpha \in \mathcal{G}}$ such that

$$
d_{G} y_{\gamma} \in(\Lambda Y)_{<\gamma} \text { and } d_{C}\left(1 \otimes x_{\alpha}\right) \in \Lambda Y \otimes(\Lambda X)_{<\alpha} .
$$

Moreover the augmentations are precisely given by $\varepsilon_{G}(Y)=0, \varepsilon_{C}(Y)=$ $\varepsilon_{C}(X)=0$, and $\varepsilon_{A}(X)=0$.

In particular, $\left(C, d_{C}, \varepsilon_{C}\right)$ is a $K S$ complex.

If $\mathcal{E}: G \rightarrow{ }^{i} C \rightarrow \rightarrow^{P} A$ is a $\Lambda$-extension, then (3.1) shows that the sequence of differential spaces

$$
0 \rightarrow\left(Q(G), Q\left(d_{G}\right)\right) \stackrel{Q(i)}{\rightarrow}\left(Q(C), Q\left(d_{C}\right)\right) \stackrel{Q(\rho)}{\rightarrow}\left(Q(A), Q\left(d_{A}\right)\right) \rightarrow 0
$$

is short exact. Hence it induces a long exact sequence

$$
\begin{aligned}
\ldots \rightarrow H^{p}(Q(G)) \stackrel{Q(i)^{*}}{\rightarrow} H^{p}(Q(C)) \stackrel{Q(\rho)^{*}}{\rightarrow} & H^{p}(Q(A)) \\
& H^{p+1}(Q(G)) \rightarrow \ldots .
\end{aligned}
$$

3.3. DefInITION. $\partial^{*}$ is called the connecting homomorphism for $\mathcal{E}$.

Now suppose $\eta:\left(R, d_{R}, \varepsilon_{R}\right) \rightarrow\left(L, d_{L}, \varepsilon_{L}\right)$ is a homomorphism, with $H^{0}(R)$ $=k=H^{0}(L)$. Consider the diagram

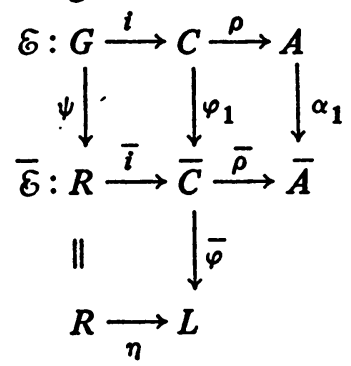

in which $(\overline{\mathscr{E}}, \bar{\varphi})$ is the minimal model for $\eta,(G, \psi)$ is the minimal model for $R$, and $\left(\mathcal{E}, \varphi_{1}\right)$ is the minimal model for $i \circ \psi$. Since ker $\rho$ is generated by $i\left(\operatorname{ker} \varepsilon_{G}\right)$, and since

$$
\bar{i} \varphi\left(\operatorname{ker} \varepsilon_{G}\right) \subset \bar{i}\left(\operatorname{ker} \varepsilon_{R}\right) \subset \operatorname{ker} \bar{\rho},
$$

$\varphi_{1}$ factors over $\rho$ to yield $\alpha_{1}$.

Note that $\psi^{*}$ and $\varphi_{1}^{*}$ are isomorphisms by definition; it follows from [8, Theorem 7.2] that, hence, so is $\alpha_{1}$. Thus we may identify $A$ with $\bar{A}$ via $\alpha_{1}$. Now collapse (3.4) to the commutative diagram

in which $\varphi=\bar{\varphi} \varphi_{1}$.

$$
\begin{array}{rlllll}
\varepsilon: G & \rightarrow & C & \stackrel{\rho}{\rightarrow} & A \\
\psi \downarrow & & \downarrow \varphi & & \\
R & \rightarrow & L & &
\end{array}
$$


Then $\mathcal{E}$ is $\Lambda$-minimal and $\psi^{*}$ and $\varphi^{*}$ are isomorphisms. By [8, Theorem 10.3] this determines $\mathcal{E}$ up to isomorphism.

3.6. Definition. $(\mathcal{E}, \psi, \varphi)$ is called the $\Lambda$-minimal $\Lambda$-model for $\eta$.

Consider the $\Lambda$-minimal $\Lambda$-model $(\mathcal{E}, \psi, \varphi)$ for $\eta$; since $G$ and $\mathcal{E}$ are minimal we have

$$
Q\left(d_{G}\right)=0 \text { and } Q\left(d_{A}\right)=0 .
$$

It follows that

$$
Q\left(d_{C}\right)=Q(i) \partial^{*} Q(\rho),
$$

where $\partial^{*}$ is the connecting homomorphism for $\mathcal{E}$.

As we have seen above, the $\Lambda$-model $(\mathcal{E}, \psi, \varphi)$ is determined by $\eta$; in particular, $\eta$ determines the model $(C, \varphi)$ for $L$. (3.7) shows that $(C, \varphi)$ is the minimal model for $L$ if and only if $\partial^{*}=0$. By the exactness of (3.2) this is equivalent to $Q(i)^{*}$ injective. But diagram (3.5) shows that

$$
Q(i)^{*}=\eta^{\#}: \pi_{\psi}^{*}(R) \rightarrow \pi_{\psi}^{*}(L) .
$$

This proves

3.8. Proposition. The model $(C, \varphi)$ for $L$ in the $\Lambda$-minimal $\Lambda$-model for $\eta$ is itself minimal if and only if $\eta^{\#}$ is injective.

4. Rational fibrations. Let $S \leadsto(A(S), d)$ be the contravariant functor from topological spaces to c.g.d.a.'s defined by Sullivan [8, Definition 15.2]. (An element $\Phi \in A^{p}(S)$ is a function which assigns to each singular $q$-simplex of $S$ a polynomial differential form with coefficients in $k$ on the standard $q$-simplex, in a way compatible with the face and degeneracy operators.)

Note that $A(\mathrm{pt})=k$, so that an inclusion $\mathrm{pt} \rightarrow S$ augments $A(S)$. By [8, Property 15.6] integration provides a natural isomorphism of graded algebras

$$
H(A(S)) \stackrel{\approx}{\rightarrow} H^{*}(S ; k) .
$$

(Cf. also [10], [13], [4] and [12].)

Fix a base point in $S$ (so that $A(S)$ is augmented) and assume $S$ is path connected (so that $H^{0}(A(S))=k$ ). Then a model for $A(S)$ will be called simply a model for $S$ and the graded space $\pi_{\psi}^{*}(A(S))$ will be denoted by $\pi_{\psi}^{*}(S)$ and called the $\psi$-homotopy space of $S$.

A continuous map $\varphi: S \rightarrow T$ (between pointed spaces) gives a homomorphism $A(\varphi): A(T) \rightarrow A(S)$ (between augmented c.g.d.a.'s); the resulting maps of cohomology and $\psi$-homotopy will be written

$$
\varphi^{*}: H^{*}(T ; k) \rightarrow H^{*}(S ; k) \text { and } \varphi^{\#}: \pi_{\psi}^{*}(T) \rightarrow \pi_{\psi}^{*}(S) .
$$

If $H^{*}(S ; \mathrm{Q})$ has finite type and $S$ is 1-connected then there are natural isomorphisms 


$$
\pi_{\psi}^{*}(S) \stackrel{\approx}{\rightarrow} \operatorname{Hom}_{\mathrm{Z}}\left(\pi_{*}(S), k\right)
$$

(cf. [12] and [8]).

Now suppose

$$
B \stackrel{\pi}{\leftarrow} E \stackrel{j}{\leftarrow} F
$$

is a sequence of base point preserving continuous maps between poind, path connected spaces, such that $\pi j(F)$ is the base point of $B$. Then

$$
A(B) \stackrel{A(\pi)}{\rightarrow} A(E) \stackrel{A(j)}{\rightarrow} A(F)
$$

is a sequence of augmented c.g.d.a.'s; denote the augmentations by $\varepsilon_{B}, \varepsilon_{E}$ id $\boldsymbol{\varepsilon}_{\boldsymbol{F}}$.

Let $(\overline{\mathcal{E}}, \bar{\varphi})$ be the minimal model for $A(\pi)$, and write

$$
\overline{\mathcal{E}}: A(B) \stackrel{\bar{i}}{\rightarrow} \bar{C} \stackrel{\bar{\rho}}{\rightarrow} \bar{A} .
$$

Because of (2.2) ker $\bar{\rho}$ is the ideal generated by $\bar{i}\left(\operatorname{ker} \varepsilon_{B}\right)$; since $A(j) A(\pi)=_{B}$ it follows that $\bar{\varphi}$ factors over $\bar{\rho}$ to yield the commutative diagram

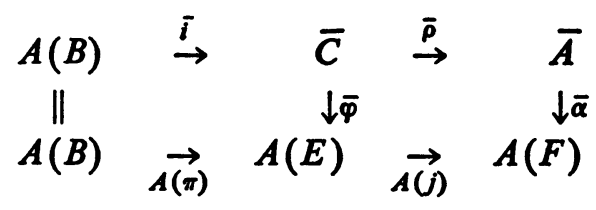

of homomorphisms of augmented c.g.d.a.'s.

4.5. DefINITION. The sequence (4.2) will be called a rational fibration if*: $H(\bar{A}) \rightarrow H(A(F))$ is an isomorphism. (Since the passage from $Q$ to is simply via $\otimes_{\mathrm{Q}} k$, this condition is independent of $k$.)

Thus (4.2) is a rational fibration if and only if $(\bar{A}, \bar{\alpha})$ is the minimal mlel for $A(F)$.

Theorem 4.6 below shows that many Serre fibrations are ratial fibrations. It was first proved in the simply connected case by Grivel [5]. or that case it was established independently a little later by J. C. Thomas.) is proved in detail in [8, Theorem 20.3] and is indeed the main goal of the notes.

4.6. THEOREM. Assume that (4.2) is a Serre fibration. Suppose further that

(i) $\pi_{1}(B)$ acts nilpotently on each $H^{p}(F ; Q), p>1$.

(ii) Either $H^{*}(F ; \mathbf{Q})$ or $H^{*}(B ; \mathbf{Q})$ is a graded space of finite type.

Then (4.2) is a rational fibration.

Now suppose $F \rightarrow^{j} E \rightarrow^{\pi} B$ is a rational fibration, and let $(\mathcal{E}, \psi, \varphi)$ behe $\Lambda$-minimal $\Lambda$-model for $A(\pi)$.

Combine diagrams (3.4) and (4.4) to obtain the commutative diagram 


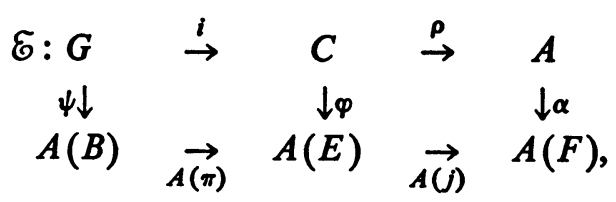

in which $\alpha=\bar{\alpha} \circ \alpha_{1}, \mathcal{E}$ is a $\Lambda$-minimal $\Lambda$-extension, and $\psi^{*}, \varphi^{*}$ and $\alpha^{*}$ are isomorphisms.

4.8. Definttion. Diagram (4.7) is called the $\Lambda$-minimal $\Lambda$-model for the rational fibration $F \rightarrow E \rightarrow B$.

Next note that, in view of (4.7), we can write the long exact sequence (3.2) in the form

$$
\ldots \rightarrow \pi_{\psi}^{p}(B) \stackrel{\pi^{*}}{\rightarrow} \pi_{\psi}^{p}(E) \stackrel{j^{*}}{\rightarrow} \pi_{\psi}^{p}(F) \stackrel{\partial^{*}}{\rightarrow} \pi_{\psi}^{p+1}(B) \rightarrow \ldots
$$

4.10. REMARK. Suppose $F \rightarrow E \rightarrow B$ is both a Serre fibration and a rational fibration, and that the isomorphisms (4.1) exist for each of $F, E$ and $B$. Then the isomorphisms (4.1) transform (4.9) to the dual of the standard long exact homotopy sequence of the fibration.

4.11. Definition. The sequence (4.9) is called the long exact $\psi$-homotopy sequence for the rational fibration. $\partial^{*}$ is called the connecting homomorphism.

4.12. PROPOSITION. The following are equivalent conditions on the rational fibration $F \rightarrow^{j} E \rightarrow^{\pi} B$ :

(i) $\partial^{*}=0$.

(ii) $\pi^{\#}$ is injective.

(iii) $j^{\#}$ is surjective.

(iv) The model for $E$ in the $\Lambda$-minimal $\Lambda$-model for the fibration is minimal.

Proof. Combine Proposition 3.8 and (4.9). Q.E.D.

The rational fibrations satisfying the conditions of Proposition 4.12 are precisely those whose $\Lambda$-minimal $\Lambda$-model is obtained by "decomposing" the minimal model of $E$, without adding any additional data. In this sense they are intrinsic to the space $E$ :

4.13. Definition. If the conditions of Proposition 4.12 hold we say $F \rightarrow E$ $\rightarrow B$ is an intrinsic rational fibration.

Now consider a rational fibration

$$
F \stackrel{j}{\rightarrow} E \stackrel{\pi}{\rightarrow} B
$$

with connecting homomorphism $\partial^{*}$. A main object of this paper is the following two theorems:

4.15. THEOREM. In the rational fibration (4.14):

(i) If $\partial^{*}$ is injective then $H^{*}(F ; k)$ is the free commutative graded algebra over $\pi_{\psi}^{*}(F): H^{*}(F ; k)=\Lambda \pi_{\psi}^{*}(F)$. 
(ii) If $\partial^{*}=0$ and $H^{p}(E ; k)=0, p>N$, then any $N+1$ cohomology classes $\gamma_{1}, \ldots, \gamma_{N+1}$ in $H^{+}(F ; k)$ have product zero: $\gamma_{1} \cdot \cdots \cdot \gamma_{N+1}=0$.

(iii) If $\operatorname{dim} H^{*}(F ; k)<\infty$, then $\partial^{*}$ is zero on elements of even degree: $\partial^{*}\left(\pi_{\psi}^{\text {even }}(F)\right)=0$.

(iv) If $\operatorname{dim} \pi_{\psi}^{*}(E)<\infty$ and $H^{p}(F ; k)=0, p>N$, then $\operatorname{dim} \pi_{\psi}^{*}(F)<\infty$, $\operatorname{dim} H^{*}(F ; k)<\infty$ and $\operatorname{dim} \pi_{\psi}^{*}(B)<\infty$.

In particular, the minimal models for $F$ and for $B$ have finitely many generators.

Proof. Apply the theorems of the next section to the $\Lambda$-minimal $\Lambda$-model of (4.14). Thus Theorem 5.2 yields (i), Corollary 5.10 gives (ii), Remark 5.17 with Theorem 5.16 implies (iii) and (iv) follows from Theorem 5.23. Q.E.D.

4.16. Corollary. For a rational fibration in which $\operatorname{dim} H^{*}(F ; k)<\infty$, the sequence

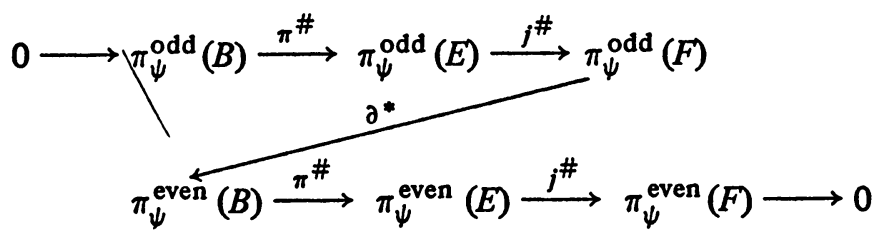

is exact.

In particular,

$$
\operatorname{dim} \pi_{\psi}^{\text {even }}(F)<\operatorname{dim} \pi_{\psi}^{\text {even }}(E) .
$$

Hence if the minimal model for $E$ has only odd generators, the same is true for the minimal model for $F$.

4.17. THEOREM. Let $\varphi: S \rightarrow T$ be a continuous map between path connected spaces, preserving base points. Assume

(i) $H^{p}(T ; k)=0, p>N$, and

(ii) $\varphi^{\#}: \pi_{\psi}^{*}(T) \rightarrow \pi_{\psi}^{*}(S)$ is surjective.

Then the product of any $N+1$ cohomology classes $\gamma_{1}, \ldots, \gamma_{N+1}$ in $\mathrm{H}^{+}(S$; $k$ ) is zero: $\gamma_{1} \cdots \cdots \cdot \gamma_{N+1}=0$.

Proof. Apply Theorem 5.9. Q.E.D.

4.18. Remark. In view of Theorem 4.6 and the isomorphisms (4.1), Theorems 4.15 and 4.17 imply Theorems 1.4 and 1.5.

5. The algebraic theorems. Consider first a $\Lambda$-extension

$$
\varepsilon: G \stackrel{i}{\rightarrow} C \stackrel{\rho}{\rightarrow} A
$$

which we write in the form (3.1). Choose bases $\left\{y_{\gamma}\right\}_{\gamma \in \mathcal{g}}$ and $\left\{x_{\alpha}\right\}_{\alpha \in \mathcal{G}}$ as 
described at the start of $\S 3$. Use $\zeta_{G}, \zeta_{C}$ and $\zeta_{A}$ to identify $Y, Y \oplus X$, and $X$ with $Q(G), Q(C)$ and $Q(A)$.

5.2. THEOREM. Assume that $\mathcal{E}$ is minimal as a KS extension and that the connecting homomorphism, $\partial^{*}$, for $\mathcal{E}$ is injective.

Then every cocycle $\Phi$ in $\operatorname{ker} \varepsilon_{C}$ satisfies $\rho \Phi=0$. In particular, $d_{A}=0$.

Proof. We induct on the well-ordered set 9 . It is sufficient to prove the theorem is correct when $\Lambda X$ is replaced by $(\Lambda X)_{<\alpha}$, assuming it to hold when $\Lambda X$ is replaced by $(\Lambda X)_{<\alpha}$.

First note that $d_{C}\left(1 \otimes x_{\alpha}\right)$ is a cocycle of degree $>1$ in $\Lambda Y \otimes(\Lambda X)_{<\alpha}$; hence by our induction hypothesis $d_{C}\left(1 \otimes x_{\alpha}\right) \in \Lambda^{+} Y \otimes(\Lambda X)_{<\alpha}$. Thus

$$
d_{\Lambda} x_{\alpha}=\rho\left(d_{C}\left(1 \otimes x_{\alpha}\right)\right)=0 .
$$

It remains to show that if $\Phi \in \Lambda Y \otimes(\Lambda X)_{<\alpha}$ satisfies $\varepsilon_{C} \Phi=0$ and $d_{C} \Phi=0$ then $\rho \Phi=0$.

Extend $\zeta_{G}$ to a projection $\zeta_{G}: \Lambda Y \rightarrow Y \oplus k$ by setting $\zeta_{G}(\lambda)=\lambda, \lambda \in k$. Define a c.g.d.a. $\left((k \oplus Y) \otimes(\Lambda X)_{<\alpha}, D\right)$ by

$$
D \circ\left(\zeta_{G} \otimes \iota\right)=\left(\zeta_{G} \otimes \iota\right) \circ d_{C} .
$$

Then $D$ restricts to $Q\left(d_{G}\right)$ in $Y \otimes 1$.

Write $Y=\operatorname{Im} Q\left(d_{G}\right) \oplus Z$ and project $Y$ onto $Z$ with kernel $\operatorname{Im} Q\left(d_{G}\right)$. This defines a projection

$$
(p \otimes \iota):(k \oplus Y) \otimes(\Lambda X)_{<\alpha} \rightarrow(k \oplus Z) \otimes(\Lambda X)_{<\alpha} .
$$

Define a c.g.d.a. $\left((k \oplus Z) \otimes(\Lambda X)_{<\alpha}, d\right)$ by

$$
d \circ(p \otimes \iota)=(p \otimes \iota) \circ D \text {. }
$$

Observe that

$$
Z \cdot Z=0 \text { and } d(Z \otimes 1)=0 .
$$

Moreover, since $d_{A}=0$ in $(\Lambda X)_{<\alpha}$, it follows that $d_{C}\left(1 \otimes(\Lambda X)_{<\alpha}\right) \subset \Lambda^{+} Y$ $\otimes(\Lambda X)_{<\alpha}$. Hence

$$
\operatorname{Im} d \subset Z \otimes(\Lambda X)_{<\alpha^{\circ}}
$$

It follows from (5.4) and (5.5) that

$$
d\left(Z \otimes(\Lambda X)_{<\alpha}\right)=0 .
$$

Finally, regard $H\left(Y, Q\left(d_{G}\right)\right)$ as a subspace of $Z$. Then we have (because of (5.5))

$$
d\left(1 \otimes x_{\beta}\right)=\partial^{*} x_{\beta} \otimes 1+\Omega_{\beta}, \quad \beta<\alpha,
$$

where $\Omega_{\beta} \in Z \otimes\left(\Lambda^{+} X\right)_{<\beta}$.

We next show that

$$
(\operatorname{ker} d) \cap\left(1 \otimes(\Lambda X)_{<\alpha}\right)=k .
$$


It is enough to show (via induction on $g$ ) that if for some $\gamma<\alpha,(\operatorname{ker} d) \cap(1$ $\left.\otimes(\Lambda X)_{<\gamma}\right)=k$, then $(\operatorname{ker} d) \cap\left(1 \otimes(\Lambda X)_{<\gamma}\right)=k$.

Let $\Psi \in(\Lambda X)_{<\gamma}$ satisfy $d(1 \otimes \Psi)=0$. Write

$$
(k \oplus Z) \otimes(\Lambda X)_{<\gamma}=(k \oplus Z) \otimes(\Lambda X)_{<\gamma} \otimes \Lambda x_{\gamma},
$$

and write

$$
1 \otimes \Psi=1 \otimes \sum_{j=0}^{m}\left(\Psi_{j} \otimes x_{\gamma}^{j}\right), \quad \Psi_{j} \in(\Lambda X)_{<\gamma}, \Psi_{m} \neq 0 .
$$

It follows from the definitions that $d\left(1 \otimes 1 \otimes x_{\gamma}\right) \in(k \oplus Z) \otimes(\Lambda X)_{<\gamma}$. Hence we can write

$$
\begin{aligned}
0= & d(1 \otimes \Psi)=d\left(1 \otimes \Psi_{m}\right) \otimes x_{\gamma}^{m} \\
& +\sum_{j=0}^{m-1}\left[d\left(1 \otimes \Psi_{j}\right) \pm(j+1)\left(1 \otimes \Psi_{j}\right) d\left(1 \otimes 1 \otimes x_{\gamma}\right)\right] \otimes x_{\gamma}^{j} .
\end{aligned}
$$

This shows that $d\left(1 \otimes \Psi_{m}\right)=0$, and so by our induction hypothesis $\Psi_{m}$ is a scalar $\lambda$.

Were $m>0$ we would also have

$$
d\left(1 \otimes \Psi_{m-1}\right) \pm m \lambda d\left(1 \otimes 1 \otimes x_{\gamma}\right)=0 .
$$

Using (5.7) we would then conclude that

$$
\partial^{*} x_{\gamma} \otimes 1 \in \partial^{*}\left(X_{<\gamma}\right) \otimes 1+Z \otimes\left(\Lambda^{+} X\right)_{<\gamma}
$$

This would imply $\partial^{*} x_{\gamma} \in \partial^{*}\left(X_{<\gamma}\right)$, and contradict the injectivity of $\partial^{*}$.

It follows that $m=0$ and so $\Psi=\Psi_{m} \in k$. (5.8) is thereby established.

Finally, suppose $\Phi \in \Lambda Y \otimes(\Lambda X)_{<\alpha}$ is a $d_{C}$-cocycle such that $\varepsilon_{C} \Phi=0$. Then

$$
\left(p \zeta_{G} \otimes \iota\right) \Phi=\Phi_{1}+1 \otimes \rho \Phi,
$$

where $\Phi_{1} \in Z \otimes(\Lambda X)_{<\alpha}$ and $\rho \Phi \in\left(\Lambda^{+} X\right)_{<\alpha^{*}}$ By (5.6) $d \Phi_{1}=0$ and so $d(1 \otimes \rho \Phi)=0$. Since $\rho \Phi \in\left(\Lambda^{+} X\right)_{<\alpha},(5.8)$ shows that $\rho \Phi=0$. Q.E.D.

Now we rely on Theorem 5.2 to establish our next result. If one skips to Corollary 5.10 it becomes clear that it deals with the opposite extreme: the case $\partial^{*}=0$.

5.9. THEOREM. Assume $\eta:\left(R, d_{R}, \varepsilon_{R}\right) \rightarrow\left(L, d_{L}, \varepsilon_{L}\right)$ is a homomorphism of augmented c.g.d.a.'s. Assume that

(i) $H^{0}(R)=H^{0}(L)=k$.

(ii) $H^{p}(R)=0, p>N$, where $N$ is some fixed integer $>0$.

(iii) $\eta^{\#}: \pi_{\psi}^{*}(R) \rightarrow \pi_{\psi}^{*}(L)$ is surjective.

Then the product of any $N+1$ cohomology classes $\gamma_{1}, \ldots, \gamma_{N+1} \in H^{+}(L)$ is zero: $\gamma_{1} \cdot \cdots \cdot \gamma_{N+1}=0$.

Proor. Let 


$$
\begin{array}{rlll}
\mathcal{E}: G & \stackrel{i}{\rightarrow} & C & \stackrel{\rho}{\rightarrow} A \\
\psi \downarrow & & \downarrow \varphi \\
R & \rightarrow & L &
\end{array}
$$

be the $\Lambda$-minimal $\Lambda$-model for $\eta$. Then we can identify $Q(i)^{*}: Q(G) \rightarrow$ $H(Q(C))$ with $\eta^{\#}$. Hence $Q(i)^{*}$ is surjective. It follows from the exactness of (3.2) that $Q(\rho)^{*}=0$ and so the connecting homomorphism $\partial^{*}$ is injective.

Since $\mathcal{E}$ is a $K S$ extension we can write $C=G \otimes A$. Moreover, since $(G, \psi)$ is the minimal model for $H(R)$, and $H^{0}(R)=k$, we have ker $\varepsilon_{G}=$ $G^{+}$. Thus Theorem 5.2 shows that every cocycle in $C$ is contained in $G^{+} \otimes A$.

In particular, because $\varphi^{*}$ is an isomorphism, there are cocycles $z_{i} \in G^{+} \otimes$ $A$ such that $\varphi z_{i}$ represents $\gamma_{i}, i=1, \ldots, N+1$.

Finally choose a subspace $I^{N} \subset G^{N}$ so that $G^{N}=I^{N} \oplus\left(\operatorname{ker} d_{G}\right)^{N}$. Let $I^{p}=0, p<N$ and $I^{p}=G^{p}, p>N$. Let $\pi: G \rightarrow G / I$ denote the projection and make $G / I$ into a c.g.d.a. $(G / I, d)$ by setting $d \pi=\pi d_{G}$. Then $\pi^{*}$ is an isomorphism.

Moreover, we can define a c.g.d.a. $(G / I \otimes A, D)$ by requiring that $(\pi \otimes$ ı) $d_{C}=D(\pi \otimes \imath)$. Since $\pi^{*}$ is an isomorphism, [8, Theorem 7.1] shows that $(\pi \otimes \iota)^{*}$ is an isomorphism.

But note that $(\pi \otimes \imath) z_{i} \in(G / I)^{+} \otimes A$. Since $(G / I)^{p}=0, p>N$, we conclude that

$$
(\pi \otimes \imath) z_{1} \cdots \cdots(\pi \otimes \imath) z_{N+1}=0 .
$$

Since $(\pi \otimes \ell)^{*}$ and $\varphi^{*}$ are isomorphisms and $\varphi z_{i}$ represents $\gamma_{i}$, the theorem follows. Q.E.D.

5.10. Corollary. Let $G \rightarrow^{i} C \rightarrow^{\rho} A$ be a $\Lambda$-extension such that $H^{0}(G)=$ $H^{0}(C)=k$ and $\partial^{*}=0$. Assume $H^{p}(C)=0, p>N$. Then $H^{0}(A)=k$ and any $N+1$ cohomology classes $\gamma_{1}, \ldots, \gamma_{N+1}$ in $H^{+}(A)$ satisfy $\gamma_{1} \cdots \cdots \gamma_{N+1}$ $=0$.

Proof. It follows from [8, Theorem 2.2] that the minimal model for $C$ is generated by a space isomorphic with $H(Q(C))$; since $H^{0}(C)=k$ we obtain $H^{0}(Q(C))=0$. Since $\partial^{*}=0$ we conclude by exactness that $H^{0}(Q(A))=0$. A second application of [8, Theorem 2.2] shows that $H^{0}(A)=k$.

Moreover, because $\partial^{*}=0, \rho^{*}$ is surjective. Thus we can apply the theorem to $\rho: C \rightarrow A$ to obtain the corollary. Q.E.D.

5.11. Corollary. Suppose $\eta: R \rightarrow L$ satisfies the hypotheses of Theorem 5.9, and $\varphi:(\Lambda Z, d) \rightarrow\left(L, d_{L}\right)$ is the minimal model of $L$. Assume $Z_{1} \subset Z$ generates a d-stable ideal, $I$. 
Then any $N+1$ cohomoloyy classes $\gamma_{1}, \ldots, \gamma_{N+1}$ in $H^{+}((\Lambda Z) / I)$ satisfy $\gamma_{1} \cdot \cdots \cdot \gamma_{N+1}=0$.

Proof. Let $\pi: \Lambda Z \rightarrow(\Lambda Z) / I$ be the projection. Write $(\Lambda Z) / I=\Lambda\left(Z / Z_{1}\right)$ and observe that this is a minimal $K S$ complex. Since $\Lambda Z$ is also, we may write

$$
\pi^{\#}=Q(\pi): Z \rightarrow Z / Z_{1} .
$$

In particular, $\pi^{\#}$ is surjective. Hence so is $(\pi \circ \eta)^{\#}=\pi^{\#} \circ \eta^{\#}$. Now apply Theorem 5.9 to $\pi \circ \eta: R \rightarrow(\Lambda Z) / I$. Q.E.D.

Again suppose $\eta: R \rightarrow L$ satisfies the hypotheses of Theorem 5.9, and $(\Lambda Z, d)$ is the minimal model for $L$. Let $\left\{z_{\gamma}\right\}_{\gamma \in \mathcal{X}}$ be a well-ordered homogeneous basis for $Z$ such that

$$
d z_{\gamma} \in(\Lambda Z)_{<\gamma}, \quad \gamma \in \mathcal{K} .
$$

Then for each $\gamma$ we have the $\Lambda$-minimal $\Lambda$-extension

$$
\left((\Lambda Z)_{<\gamma}, d\right) \rightarrow(\Lambda Z, d) \rightarrow\left((\Lambda Z)_{>\gamma}, \bar{d}\right),
$$

and $\bar{d} z_{\gamma}=0$.

5.12. CoROLlary. The class $\left[z_{\gamma}\right]$ represented by $z$ in $(\Lambda Z)_{>_{\gamma}}$ satisfies $\left[z_{\gamma}\right]^{N+1}=0$. Thus we can write $z_{\gamma}^{N+1}=d \Phi+\Psi$, where $\Psi$ is in the ideal generated by $\boldsymbol{Z}_{<\boldsymbol{r}}$.

Proof. Apply Corollary 5.11. Q.E.D.

5.13. CoRollary. Suppose that the hypotheses of Corollary 5.11 hold and that, in addition, $\operatorname{dim} Z / Z_{1}<\infty$. Then

$$
\operatorname{dim} H((\Lambda Z) / I)<\infty .
$$

Proof. Write $(\Lambda Z) / I=\Lambda\left(Z / Z_{1}\right)$, and let $\bar{z}_{1}, \ldots, \bar{z}_{n}$ be a well-ordered homogeneous basis of $Z / Z_{1}$ such that

$$
\bar{d} \bar{z}_{i} \in \Lambda\left(\bar{z}_{1}, \ldots, \bar{z}_{i-1}\right), \quad 1<i<n .
$$

Consider the composite projection

$$
\Lambda Z \rightarrow(\Lambda Z) / I \rightarrow \Lambda\left(\bar{z}_{i}, \ldots, \bar{z}_{n}\right),
$$

where the second projection has kernel generated by $\bar{z}_{1}, \ldots, \bar{z}_{i-1}$.

We can apply Corollary 5.11 to this composite to conclude that

$$
\left[\bar{z}_{i}\right]^{N+1}=0 \text { in } H\left(\Lambda\left(\bar{z}_{i}, \ldots, \bar{z}_{n}\right)\right), \quad 1<i<n .
$$

It follows now from [7, Proposition 1] that $\operatorname{dim} H((\Lambda Z) / I)<\infty$. Q.E.D.

It would be more satisfying if in Theorem 5.9 we could weaken the hypothesis on $R$ to resemble the conclusion on $L$ or alternatively strengthen 
the conclusion to $\operatorname{dim} H(L)<\infty$. Neither of these is possible as the next two examples show:

5.14. EXAMPLE. Consider the $\Lambda$-minimal $\Lambda$-extension

$$
\Lambda(x, y) \rightarrow \Lambda(x, y, u, v) \rightarrow \Lambda(u, v)
$$

where $\operatorname{deg} x=3, \operatorname{deg} y=2, \operatorname{deg} u=4$ and $\operatorname{deg} v=3$, and the differential $d$ in $\Lambda(x, y, u, v)$ is given by

$$
d x=d y=0, d u=x y, d v=y^{2} .
$$

The differentials in $\Lambda(x, y)$ and $\Lambda(u, v)$ are consequently zero.

An easy computation checks that the cocycles $x u^{k}$ and $y u^{k}+k x u^{k-1} v$ $(k>0)$ represent cohomology classes which form a basis of $H(\Lambda(x, y, u, v))$. It follows easily that

$$
H^{+}(\Lambda(x, y, u, v)) \cdot H^{+}(\Lambda(x, y, u, v))=0 .
$$

On the other hand, in $H(\Lambda(u, v))=\Lambda(u, v)$ no power of $u$ is zero. Thus we cannot weaken the hypothesis on $R$.

5.15. EXAMPLE. Consider the inclusion $H^{*}\left(S^{3} ; k\right) \rightarrow H^{*}\left(S^{3} \vee S^{3} ; k\right)$ (induced by the collapse of one sphere to a point) as a homomorphism of c.g.d.a.'s (with zero differential). Form the corresponding $\Lambda$-minimal $\Lambda$-model

$$
H^{*}\left(S^{3}\right) \stackrel{i}{\rightarrow} R \stackrel{\rho}{\rightarrow} L .
$$

It is trivial that $Q(i)^{*}$ is injective and so $Q(\rho)^{*}=\rho^{\#}$ is surjective. Since $\operatorname{dim} H(R)<\infty$, Theorem 5.9 applies and shows in fact that the product of any four cohomology classes in $H^{+}(L)$ is zero.

But we cannot strengthen the theorem and conclude $\operatorname{dim} H(L)<\infty$. Indeed, there is an obvious spectral sequence converging from $H^{*}\left(S^{3}\right) \otimes$ $H(L)$ to $H\left(S^{3} \vee S^{3}\right)$; were $\operatorname{dim} H(L)<\infty$ we could conclude that the Euler characteristic of $S^{3} \vee S^{3}$ was the product of the Euler characteristics of $S^{3}$ and of $H(L)$. This would yield

$$
0=0 \cdot \chi_{H(L)}=\chi_{S^{3} \vee S^{3}}=-1,
$$

which is a contradiction.

Next consider a $\Lambda$-extension $\varepsilon: G \rightarrow^{i} C \rightarrow^{p} A$ and, as at the beginning of this section, write it in the form

$$
\Lambda Y \stackrel{i}{\rightarrow} \Lambda Y \otimes \Lambda X \stackrel{\rho}{\rightarrow} \Lambda X .
$$

Use $\zeta_{G}, \zeta_{C}$ and $\zeta_{A}$ to identify $Y$ with $Q(G), Y \oplus X$ with $Q(C)$, and $X$ with $Q(A)$. Assume $\left\{x_{\alpha}\right\}_{\alpha \in g}$ is a well-ordered homogeneous basis for $X$ such that

$$
d_{C}\left(1 \otimes x_{\alpha}\right) \in \Lambda Y \otimes(\Lambda X)_{<\alpha}, \quad \alpha \in \mathscr{g} .
$$

Then for each $\alpha$ we have the $\Lambda$-extension

$$
(\Lambda X)_{<\alpha} \rightarrow \Lambda X \rightarrow(\Lambda X)_{>\alpha} \text {. }
$$


We will denote by $\left[x_{\alpha}\right]$ the class in $H\left((\Lambda X)_{>\alpha}\right)$ represented by $x_{\alpha^{*}}$.

5.16. THEOREM. Suppose that $\mathcal{E}$ is $\Lambda$-minimal and satisfies

(i) $H^{0}(C)=k$, and

(ii) for each $\alpha \in G$ there is an $n_{\alpha}$ such that $\left[x_{\alpha}\right]^{n_{\alpha}}=0$.

Then $A^{0}=k$ and the connecting homomorphism, $\partial^{*}$, for $\mathcal{E}$ is zero on elements of even degree.

5.17. REMARK. The hypotheses (and hence the conclusion) of Theorem 5.16 are implied by the following assumptions on $\mathcal{E}$; it is $\Lambda$-minimal, $H^{0}(A)=k$ $=H^{\circ}(C)$, and $\operatorname{dim} H(A)<\infty$.

Indeed, simply apply Corollary 5.12 with $R=L=A$.

5.18. COROLLARY. Under the hypotheses of the theorem, the sequence

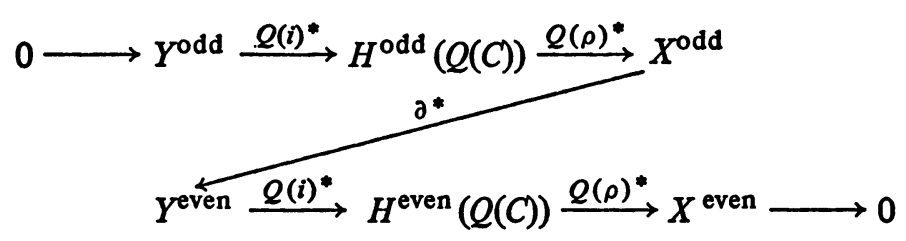

is exact.

5.19. CoRollary. Suppose E satisfies the hypotheses of the theorem, and that $\zeta_{C}^{*}: H^{\text {even }}(C) \rightarrow H^{\text {even }}(Q(C))$ is surjective. Then

$$
\zeta_{A}^{*}: H^{\text {even }}(A) \rightarrow X^{\text {even }}
$$

is surjective.

PRoof. Observe that $\zeta_{A}^{*} \rho^{*}=Q(\rho)^{*} \zeta_{C}^{*}$, and that $Q(\rho)^{*}$ is surjective by Corollary 5.18. Q.E.D.

5.20. Proof of TheOREM 5.16. Since $\mathcal{E}$ is a minimal $K S$ extension and $H^{0}(C)=k,[8$, Corollary 3.10$]$ shows that

$$
H(A) \simeq \Lambda X^{0} \otimes H\left(\Lambda X^{+}\right) \text {. }
$$

If $x_{\alpha}$ had degree zero then we could write

$$
x_{\alpha}^{n_{\alpha}}+\Phi=d_{A} \Psi
$$

where $\Phi$ and $x_{\alpha}^{n_{\alpha}}$ were linearly independent. Hence $\operatorname{deg} \Phi=0$ and so $x_{\alpha}$, $\Phi \in \Lambda X^{0}$, which contradicts the above isomorphism.

It follows that $A$ is connected, $A^{0}=k$.

Now we show by induction on $I$ that $\partial^{*} x_{\alpha}=0$ if $x_{\alpha}$ has even degree. It is enough to show this for some fixed $\alpha$, assuming the result to hold for all $\beta<\alpha$.

Write $C=\Lambda Y \otimes(\Lambda X)_{<\alpha} \otimes(\Lambda X)_{>\alpha}$; this defines a $\Lambda$-extension 


$$
\Lambda Y \otimes(\Lambda X)_{<\alpha} \stackrel{j}{\rightarrow} \Lambda Y \otimes(\Lambda X)_{<\alpha} \otimes(\Lambda X)_{>\alpha} \stackrel{\pi}{\rightarrow}(\Lambda X)_{>\alpha}
$$

Assume $\operatorname{deg} x_{\alpha}=2 n$. Then by hypothesis $\partial^{*}$ is zero in $X_{<\alpha}^{2 n}$ and so

$$
0 \rightarrow Y^{2 n+1} \rightarrow H\left(Y \oplus X_{<\alpha}, Q\left(d_{C}\right)\right)
$$

is exact. It follows that it is sufficient to prove $\bar{\partial}^{*} x_{\alpha}=0$, where $\bar{\partial}^{*}$ is the connecting homomorphism for (5.21).

Next, form the $\Lambda$-minimal $\Lambda$-model of $j$ (possible because $H^{0}(C)=k$ and so $H^{0}\left(\Lambda Y \otimes(\Lambda X)_{<\alpha}\right)=k$ too). Recall that the fibre of this $\Lambda$-extension can be identified with $(\Lambda X)_{>\alpha}$ because (5.21) is a minimal $K S$ extension [8, Theorem 7.2].

Hence the $\Lambda$-minimal $\Lambda$-model of $j$ takes the form

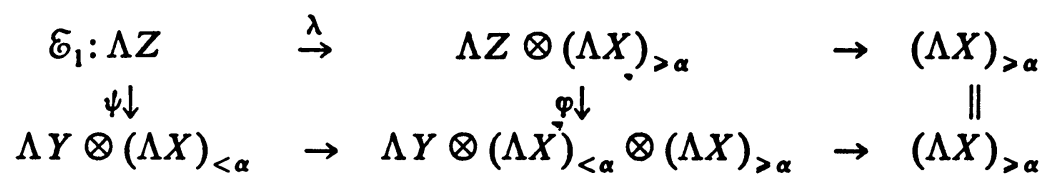

in which the upper row is a $\Lambda$-minimal $\Lambda$-extension, and $\psi^{*}$ and $\varphi^{*}$ are isomorphisms.

In particular [8, Theorem 7.1], $Q(\psi)^{*}$ and $Q(\varphi)^{*}$ are isomorphisms. Thus the diagram above identifies $\bar{\partial}^{*}$ with the connecting homomorphism $\left(\partial_{1}\right)^{*}$ for $\mathcal{E}_{1}$, and so we have only to show that

$$
\left(\partial_{1}\right)^{*} x_{\alpha}=0
$$

Denote $\left(\partial_{1}\right)^{*} x_{\alpha}$ by $u_{\alpha}$.

Assume (5.22) is wrong. As in the proof of Theorem 5.2 divide $\Lambda Z \otimes$ $(\Lambda X)_{>\alpha}$ by $\Lambda^{+} Z \cdot \Lambda^{+} Z \otimes(\Lambda X)_{>\alpha}$ to obtain a c.g.d.a. $(k \oplus Z) \otimes(\Lambda X)_{>\alpha} \cdot$ Since $\Lambda Z$ is minimal the induced differential in $Z \otimes 1$ is zero. Hence we can further divide out by $Z_{1} \otimes(\Lambda X)_{>\alpha}$ where $Z_{1} \subset Z$ is a graded space such that

$$
Z=Z_{1} \oplus\left(u_{\alpha}\right) \text {. }
$$

Because $x_{\alpha}$ has even degree $2 n, \operatorname{deg} u_{\alpha}=2 n+1$ and so the resulting factor c.g.d.a. has the form $\left(\Lambda u_{\alpha} \otimes(\Lambda X)_{>\alpha}, D\right)$, and $\Lambda u_{\alpha}$ is the exterior algebra over $u_{\alpha}$. In particular, $D$ has the form $D\left(u_{\alpha} \otimes 1\right)=0$ and

$$
D(1 \otimes \Phi)=u_{\alpha} \otimes \theta(\Phi)+1 \otimes d \Phi,
$$

where $d$ is the induced differential in $(\Lambda X)_{>\alpha}$ and $\theta$ is a derivation of degree $-2 n$ such that $\theta d=d \theta$.

Now in $(\Lambda X)_{>\alpha}$ we have $d x_{\alpha}=0$, and so $D\left(1 \otimes x_{\alpha}\right)=u_{\alpha} \otimes \theta\left(x_{\alpha}\right)$. Since $A$ is connected $\theta\left(x_{\alpha}\right)$ is a scalar, and since $u_{\alpha}=\left(\partial_{1}\right)^{*} x_{\alpha}$ we conclude $\theta\left(x_{\alpha}\right)=$ 1. Thus

$$
D\left(1 \otimes x_{\alpha}\right)=u_{\alpha} \otimes 1 .
$$

Furthermore, by hypothesis, $x_{\alpha}^{n_{\alpha}}$ is a coboundary in $(\Lambda X)_{>\alpha}$. Choose the least $m>1$ such that $x_{\alpha}^{m}=d \Phi$, some $\Phi \in(\Lambda X)_{>\alpha}$. Then 


$$
\begin{aligned}
0 & =D^{2}(1 \otimes \Phi)=D\left(1 \otimes x_{\alpha}^{m}+u_{\alpha} \otimes \theta \Phi\right) \\
& =m u_{\alpha} \otimes x_{\alpha}^{m-1}-u_{\alpha} \otimes d \theta \Phi .
\end{aligned}
$$

This shows that $x_{\alpha}^{m-1}=d(\theta \Phi / m)$, which contradicts the minimality of $m$, when $m>1$.

But if $m=1$ we have $x_{\alpha}=d \Phi$, which contradicts the minimality of $\left((\Lambda X)_{>\alpha}, d\right)$. We have thus arrived at a contradiction, and so (5.22) must be correct. This completes the proof. Q.E.D.

Again consider a $\Lambda$-extension $\mathcal{E}: G \rightarrow \rightarrow^{i} C \rightarrow{ }^{\rho} A$.

5.23. THEOREM. Assume that $\mathcal{E}$ is $\Lambda$-minimal. Suppose that

(i) $\operatorname{dim} H(Q(C))<\infty$.

(ii) $H^{0}(A)=k$ and $H^{p}(A)=0, p>N$.

Then $\operatorname{dim} Q(A)<\infty, \operatorname{dim} Q(G)<\infty$ and $\operatorname{dim} H(A)<\infty$.

Proof. As previously, write $\mathcal{E}$ in the form $\Lambda Y \rightarrow \Lambda Y \otimes \Lambda X \rightarrow \Lambda X$, and identify $Y=Q(G), X=Q(A), Y \oplus X=Q(C)$.

Since $\operatorname{ker} \partial^{*}=\operatorname{Im} Q(\rho)^{*}$ and $\operatorname{dim} H(Q(C))<\infty$, we conclude that $\operatorname{dim} \operatorname{ker} \partial^{*}<\infty$.

Choose a well-ordered homogeneous basis $\left\{x_{\alpha}\right\}_{\alpha \in g}$ for $X$ so that $d_{C}(1 \otimes$ $\left.x_{\alpha}\right) \in \Lambda Y \otimes(\Lambda X)_{<\alpha}$. It is an easy exercise to do so, so that in addition a subset of the $x_{\alpha}$ 's is a basis for ker $\partial^{*}$. Thus we may assume that $x_{\alpha_{1}}, \ldots, x_{\alpha_{m}}$ is a basis for ker $\partial^{*}$.

An easy induction argument shows that then there is an additional finite subset of the $x$ 's, $x_{\alpha_{m+1}}, \ldots, x_{\alpha_{n}}$, so that $\Lambda Y \otimes \Lambda\left(x_{\alpha_{1}}, \ldots, x_{\alpha_{n}}\right)$ is $d_{C}$-stable. Thus we can reorder $\mathscr{G}$ so that $\alpha_{1}, \ldots, \alpha_{n}$ are the first $n$ elements of $\mathscr{G}$ and still retain the condition that $d_{C}\left(1 \otimes x_{\alpha}\right) \in \Lambda Y \otimes(\Lambda X)_{<\alpha}$. We do this, and forget the old ordering.

Let $Z$ be the span of $x_{\alpha_{1}}, \ldots, x_{\alpha_{n}}$ and consider the $\Lambda$-extension

$$
\Lambda Y \otimes \Lambda Z \rightarrow(\Lambda Y \otimes \Lambda Z) \otimes(\Lambda X)_{>\alpha_{n}} \rightarrow(\Lambda X)_{>\alpha_{n}} .
$$

Its connecting homomorphism, $\bar{\partial}^{*}$, may be identified with the composite

$$
X_{>\alpha_{n}} \stackrel{\partial^{*}}{\rightarrow} Y \stackrel{\text { proj }}{\rightarrow} Y / \partial^{*}(Z) \text {. }
$$

Since $Z \supset$ ker $\partial *$ it follows that $\bar{\partial}^{*}$ is injective.

Now Theorem 5.2 applies and shows that the differential $\bar{d}_{A}$ in $(\Lambda X)_{>\alpha_{n}}$ is zero; i.e.

$$
H\left((\Lambda X)_{>\alpha_{n}}\right)=(\Lambda X)_{>\alpha_{n}} .
$$

On the other hand, consider the $\Lambda$-minimal $\Lambda$-extension

$$
\Lambda Z \rightarrow \Lambda X \rightarrow(\Lambda X)_{>\alpha_{n}}
$$

Since $\Lambda X$ is a minimal complex (because $\mathcal{E}$ was assumed $\Lambda$-minimal) it follows that the connecting homomorphism for this extension is zero. Thus all 
the hypotheses of Corollary 5.10 are satisfied and we conclude that the product of $N+1$ elements in $H^{+}\left((\Lambda X)_{>a_{n}}\right)$ is zero. In view of (5.24) we obtain that the product of $N+1$ elements in $X_{>\alpha_{n}}$ is zero in $(\Lambda X)_{>\alpha_{n}}$.

It follows that $\operatorname{dim} X_{>\alpha_{n}}<N$, and so

$$
\operatorname{dim} X=\operatorname{dim} Z+\operatorname{dim} X_{>\alpha_{n}}<n+N<\infty .
$$

Hence $\Lambda X$ has finite type and so then does $H(\Lambda X)=H(A)$. Since $H^{p}(A)=$ $0, p>N$, we obtain $\operatorname{dim} H(A)<\infty$.

Finally, since

$$
X \stackrel{\partial^{*}}{\rightarrow} Y^{Q(i)^{*}} \rightarrow H(Q(C))
$$

is exact, and $\operatorname{dim} X<\infty, \operatorname{dim} H(Q(C))<\infty$, we conclude that $\operatorname{dim} Y<$ $\infty$ Q.E.D.

6. Spaces of finite rank. A path connected space $S$ is said to have finite rank if $\operatorname{dim} \pi_{\psi}^{*}(S)<\infty$. In this case we call

$$
\chi_{\pi}(S)=\sum_{p}(-1)^{p} \operatorname{dim} \pi_{\psi}^{p}(S)
$$

the homotopy Euler characteristic of $S$. If $\operatorname{dim} H^{*}(S ; k)<\infty$ then [7, Theorem 1] shows that $\chi_{\pi}(S)<0$.

Assume now that

$$
F \stackrel{j}{\rightarrow} E \stackrel{\pi}{\rightarrow} B
$$

is a rational fibration. If all of $F, E, B$ have finite rank then the exact $\psi$-homotopy sequence for (6.1) (cf. (4.9)) shows that

$$
\chi_{\pi}(E)=\chi_{\pi}(B)+\chi_{\pi}(F) \text {. }
$$

6.3. THEOREM. Suppose that $F \rightarrow^{j} E \rightarrow^{\pi} B$ is a rational fibration. Assume $E$ has finite rank, and that for integers $M, N>0$,

$$
H^{p}(B ; k)=0, \quad p>M \text { and } H^{q}(F ; k)=0, \quad q>N .
$$

Then

(i) $B$ and $F$ also have finite rank.

(ii) $H^{*}(B ; k), H^{*}(F ; k)$ and $H^{*}(E ; k)$ have finite dimension.

(iii) $\chi_{\pi}(B)<0, \chi_{\pi}(F)<0$ and $\chi_{\pi}(E)<0$.

Proof. It follows from Theorem 4.15(iv) that $B$ and $F$ have finite rank and that $\operatorname{dim} H^{*}(F ; k)<\infty$. Since $B$ has finite rank its minimal model is finitely generated and so of finite type. Hence also $H^{*}(B ; k)$ has finite type. It follows from our hypothesis that $\operatorname{dim} H^{*}(B ; k)<\infty$.

Let $\Lambda Y \rightarrow \Lambda Y \otimes \Lambda X \rightarrow \Lambda X$ be the $\Lambda$-minimal $\Lambda$-model of the rational fibration. As in the proof of Theorem 5.9 define a projection of $\Lambda Y$ onto a 
c.g.d.a. $U$ such that $U^{p}=0, p>M$, and the projection induces an isomorphism of cohomology.

Extend the projection to a projection $\Lambda Y \otimes \Lambda X \rightarrow U \otimes \Lambda X$ which also induces a cohomology isomorphism. Filter $U \otimes \Lambda X$ by the ideals $\Sigma_{j>p} U^{\prime} \otimes$ $\Lambda X$ to obtain a spectral sequence converging to $H^{*}(E ; k)$. Its $E_{1}$-term is $U \otimes H^{*}(F ; k)$ and so $E_{1}^{p, q}=0, p+q>M+N$. Thus $H^{r}(E ; k)=0, r>$ $M+N$.

But because $\pi_{\psi}^{*}(E)$ is finite dimensional, $H^{*}(E ; k)$ has finite type; hence $\operatorname{dim} H^{*}(E ; k)<\infty$.

The rest of the theorem follows from [7, Theorem 1] and (6.2). Q.E.D.

6.5. CoROLlary. Suppose the hypotheses of Theorem 6.3 hold and that $\chi_{\pi}(E)=0$. Then

$$
\chi_{\pi}(F)=\chi_{n}(B)=0,
$$

and there is an isomorphism of graded $H^{*}(B ; k)$ modules

compatible with $\pi^{*}$ and $j^{*}$.

$$
H^{*}(E ; k) \simeq H^{*}(B ; k) \otimes H^{*}(F ; k)
$$

In particular, $\pi^{*}$ is injective and $j^{*}$ is surjective.

Proof. It follows from Theorem 6.3(iii) and (6.2) that $\chi_{*}(F)=\chi_{\pi}(B)=0$. Hence by [7, Theorem 1$] H^{*}(B ; k)$ and $H^{*}(F ; k)$ are evenly graded.

In particular, $H^{1}(B ; k)=H^{1}(F ; k)=0$ and so the minimal models of $B$ and of $F$ contain no elements of degree 1.

Now let $\Lambda Y \rightarrow \Lambda Y \otimes \Lambda X \rightarrow \Lambda X$ be the $\Lambda$-minimal $\Lambda$-model for the fibration, and filter $\Lambda Y \otimes \Lambda X$ by the ideals $F^{p}=\Sigma_{j>p}(\Lambda Y)^{j} \otimes \Lambda X$. The resulting spectral sequence converges to $H^{*}(E ; k)$. Since $Y$ has no elements of degree 1 , the $E_{2}$-term is given by

$$
E_{2}^{p, q}=H^{p}(B ; k) \otimes H^{q}(F ; k) \text {. }
$$

Hence $E_{2}$ has only elements of even degree, and the spectral sequence collapses. Q.E.D.

6.6. CoROLLARY. Suppose, in addition to the hypotheses of the theorem, that $\pi_{\psi}^{\text {even }}(E)=0$. Then $\pi_{\psi}^{\text {even }}(F)=0$ and

$$
\operatorname{dim} \pi_{\psi}^{*}(E)=\operatorname{dim} \pi_{\psi}^{*}(F)-\chi_{\pi}(B) .
$$

In particular, $\operatorname{dim} \pi_{\psi}^{*}(E)>\operatorname{dim} \pi_{\psi}^{*}(F)$.

Proor. Apply Corollary 4.16 and (6.2), recalling that $\chi_{\pi}(B)<0$. Q.E.D.

6.7. Corollary. Suppose $F \rightarrow E \rightarrow B$ is a rational fibration with (6.4) holding. Assume $H^{*}(E ; k)$ is an exterior algebra on $r$ elements of odd degree, where $r=1$ or 2 .

Then $H^{*}(F ; k)$ is an exterior algebra on at most $r$ elements, all of odd degree. 
6.8. REMARK. When $r=1$ the corollary generalizes a result of Borel [2] and Spanier and Whitehead [11].

Our final application of the theory to spaces of finite rank reads

6.9. TheOREM. Assume $F \rightarrow E \rightarrow{ }^{\prime} B$ is a rational fibration such that $H^{p}(F$; $k)=0, p>N$. Suppose $E$ has finite rank and suppose $H^{*}(E ; k)=\Lambda Z$ (so that $\left.Z=\pi_{\psi}^{*}(E) !\right)$.

Then the minimal model of $F$ has the form $\left(\Lambda \hat{P} \otimes \Lambda Q \otimes \Lambda P_{1}, d_{F}\right)$, where:

(i) $\hat{P}=\hat{P}^{\text {odd }}, P_{1}=P_{1}^{\text {odd }}$ and $Q=Q^{\text {even }}$;

(ii) $\hat{P} \oplus Q=j^{\#}\left(\pi_{\psi}^{*}(E)\right)$;

(iii) $d_{F}(\hat{P} \oplus Q)=0$ and $d_{F}\left(P_{1}\right) \subset \Lambda^{+}(\hat{P} \oplus Q) \otimes \Lambda P_{1}$.

6.10. CoRollary. If $\pi_{\psi}^{\text {even }}(E)=0$ then the minimal model of $F$ is given by $\left(\Lambda \hat{P} \otimes \Lambda P_{1}, d_{F}\right)$ satisfying the conditions described above.

6.11. Remarks. (1) Spaces $E$ satisfying the hypotheses of Theorem 6.5 include products of connected Lie groups, classifying spaces, and many homogeneous spaces (e.g. odd spheres and complex Stiefel manifolds). See [6] for more examples.

(2) The theorem shows that the minimal model of $F$ can be decomposed into the $\Lambda$-extension

$$
(\Lambda \hat{P} \otimes \Lambda Q, 0) \rightarrow\left(\Lambda \hat{P} \otimes \Lambda Q \otimes \Lambda P_{1}, d_{F}\right) \rightarrow\left(\Lambda P_{1}, 0\right) .
$$

(3) The theorem also yields a $\Lambda$-extension

$$
(\Lambda \hat{P}, 0) \rightarrow\left(\Lambda \hat{P} \otimes \Lambda Q \otimes \Lambda P_{1}, d\right) \rightarrow\left(\Lambda Q \otimes \Lambda P_{1}, \bar{d}\right) .
$$

Since $H^{*}(F ; k)$ has finite dimension, [7, Corollary to Proposition 1] shows that

$$
\operatorname{dim} H\left(\Lambda Q \otimes \Lambda P_{1}\right)<\infty .
$$

In particular [7, Theorem 1], $\operatorname{dim} Q<\operatorname{dim} P_{1}$.

6.12. Proof of Theorem 6.9. Let $\mathcal{E}:\left(G, d_{B}\right) \rightarrow\left(C, d_{E}\right) \rightarrow\left(A, d_{F}\right)$ be the $\Lambda$-minimal $\Lambda$-model for the fibration. It follows from the hypotheses $H(C)=$ $H^{*}(E ; k)=\Lambda Z$ that there is a homomorphism $\varphi:(\Lambda Z, 0) \rightarrow\left(C, d_{E}\right)$ such that $\varphi^{*}$ and $Q(\varphi)^{*}$ are isomorphisms.

Define $\hat{P} \subset \pi_{\psi}^{\text {odd }}(F)$ and $Q \subset \pi_{\psi}^{\text {even }}(F)$ by

$$
\hat{P} \oplus Q=\operatorname{Im} j^{\#} \text {. }
$$

Then $Q=\pi_{\psi}^{\text {even }}(F)$ as follows from Corollary 4.16.

Now write $\mathcal{E}$ in the form $\Lambda Y \rightarrow \Lambda Y \otimes \Lambda X \rightarrow \Lambda X$ and identify $\pi_{\psi}^{*}(F)=X$. It is an easy exercise to do so, so that

$$
\varphi(Z) \cap(1 \otimes X)=1 \otimes(\hat{P} \oplus Q) .
$$

It follows that $d_{E}(1 \otimes(\hat{P} \oplus Q))=0$ and so $d_{F}(\hat{P} \oplus Q)=0$. 
Finally, choose $P_{1} \subset X^{\text {odd }}$ so that $X^{\text {odd }}=\hat{P} \oplus P_{1}$. Since $Q=X^{\text {even }}$ we have

$$
\Lambda X=\Lambda \hat{P} \otimes \Lambda Q \otimes \Lambda P_{1}
$$

Since $d_{E}(1 \otimes \hat{P})=0=d_{E}(1 \otimes Q)$ we can form the $\Lambda$-extension

$$
\Lambda Y \otimes \Lambda \hat{P} \otimes \Lambda Q \rightarrow \Lambda Y \otimes \Lambda X \rightarrow \Lambda P_{1} \text {. }
$$

Moreover, it is $\Lambda$-minimal.

Thus the connecting homomorphism, $\bar{\partial}^{*}$, for this extension is a linear map $P_{1} \rightarrow Y \oplus P \oplus Q$; in fact it is just the restriction of the connecting homomorphism $\partial *$ for $\mathcal{E}$. Since by (4.9)

it follows that $\bar{\partial}^{*}$ is injective.

$$
\operatorname{ker} \partial^{*}=\operatorname{Im} j^{\#}=\hat{P} \oplus Q,
$$

We can now apply Theorem 5.2 and conclude that the differential in $\Lambda P_{1}$ is zero; i.e.

$$
d_{F}\left(P_{1}\right) \subset \Lambda^{+}(P \oplus Q) \otimes \Lambda P_{1} \text {. Q.E.D. }
$$

7. Homogeneous spaces. We shall apply the results of $\$ 6$ to fibrings of homogeneous spaces. Recall that $E \rightarrow{ }^{\pi} B$ is a locally trivial bundle with fibre $F$ if there is an open cover $\left\{U_{\alpha}\right\}_{\alpha \in \Phi}$ of $B$ and homeomorphisms $U_{\alpha} \times F$ $\rightarrow \approx_{\pi}^{-1}\left(U_{\alpha}\right)$ which convert $\pi$ to the standard projection $U_{\alpha} \times F \rightarrow U_{\alpha}$.

Assume $E \rightarrow^{\pi} B$ is a locally trivial bundle with path connected fibre $F$. Suppose $E$ is a connected $r$-manifold, and $B$ is a $C W$ complex. A crosssection over an $m$-cell of $B$ defines an embedding $R^{m} \rightarrow E$; hence by invariance of domain $m \leqslant r$.Thus $B$ is finite dimensional of dimension $M<r$. In particular,

$$
H^{p}(B ; k)=0, \quad p>M .
$$

Next trivialize the bundle over an open $M$-cell of $B$ to obtain a homeomorphism of $R^{M} \times F$ onto an open subset of $E$. If $W$ is any proper open subset of $E$ then $H^{p}(W ; k)=0, k>r$. It follows that

$$
\begin{aligned}
H^{p}(F ; k) & =H^{M+p}\left(\mathbf{R}^{M} \times F,\left(\mathbf{R}^{M}-0\right) \times F ; k\right) \\
& =H^{M+p}(E, E-F ; k)=0,
\end{aligned}
$$

if $M+p>r$. Hence setting $N=r-M$ we find

$$
H^{p}(F ; k)=0, \quad p>N .
$$

Moreover the equation (for $y \in F$ )

$$
H^{*}(E, E-y ; k)=H^{*}\left(\mathbf{R}^{M}, \mathbf{R}^{M}-0 ; k\right) \otimes H^{*}(F, F-y ; k)
$$

yields 


$$
H^{p}(F, F-y ; k)= \begin{cases}0, & p \neq N, \\ k, & p=N .\end{cases}
$$

Next let $K$ be a closed connected subgroup of a connected Lie group $G$ and suppose $K_{C} \subset G_{C}$ are maximal compact subgroups of $K$ and $G$. By Iwasawa's theorem the inclusions $K_{C} \rightarrow K$ and $G_{C} \rightarrow G$ are homotopy equivalences. Hence the inclusion $G_{C} / K_{C} \rightarrow G / K$ is also a homotopy equivalence.

According to Cartan [3] the manifold $G_{C} / K_{C}$ has finite rank and the linear map

$$
\zeta^{*}: \sum_{j>1} H^{2 j}\left(G_{C} / K_{C}\right) \rightarrow \pi_{\psi}^{\text {even }}\left(G_{C} / K_{C}\right)
$$

is surjective. (He works with coefficients $R$ but this implies the result for any $k$. A full exposition is given in [6, Chapters 10,11$]$.) Hence $G / K$ has finite rank as well and $\zeta^{*}: H^{2 j}(G / K) \rightarrow \pi_{\psi}^{2 j}(G / K)$ is also surjective.

Again by [3]

$$
\chi_{\pi}(G / K)=\operatorname{rank} K_{C}-\operatorname{rank} G_{C} .
$$

Clearly

$$
\operatorname{dim} H^{*}(G / K ; k)<\infty .
$$

Now consider an arbitrary locally trivial bundle with total space $G / K$ :

$$
F \stackrel{j}{\rightarrow} G / K \stackrel{\pi}{\rightarrow} B
$$

and suppose $B$ a CW complex. (If $B$ is 1-connected then $F$ is path connected and a simple argument on the Serre spectral sequence shows $\operatorname{dim} H^{*}(B$; $k)<\infty$. Thus in this case it follows from Grivel [5] that (7.6) is a rational fibration.)

In general (when $B$ is not 1-connected) simply assume (7.0) is a rational fibration. Because of (7.1) and (7.2) the results of $\$ 6$ (especially Theorem 6.3 and its corollaries) apply. For instance, $B$ and $F$ must have finite rank.

In particular, we obtain

7.7. THEOREM. The following manifolds do not appear as the total space of a locally trivial bundle with connected fibre over a CW complex unless either the fibre or the base is a single point:

(i) $S^{2 k}, k>1$.

(ii) $\mathrm{CP}^{p-1}, p$ a prime.

(iii) $U(4) / U(2) \times U(2)$.

Proor. Suppose such a bundle existed and write it $F \rightarrow^{j} E \rightarrow \rightarrow^{\pi} B$, where $E$ is one of the manifolds above. Since $F$ is connected and all the possibilities for $E$ are 1-connected, $B$ is 1-connected. Hence the bundle is a rational fibration and $\$ 6$ applies. 
Moreover, all the possibilities for $E$ satisfy $\chi_{\pi}(E)=0$. Thus we can apply Corollary 6.5 to obtain

$$
\operatorname{dim} H^{*}(E ; k)=\operatorname{dim} H^{*}(B ; k) \cdot \operatorname{dim} H^{*}(F ; k) .
$$

In case (i) or case (ii) $\operatorname{dim} H^{*}(E ; k)$ is a prime; hence

$$
\text { either } \operatorname{dim} H^{+}(F ; k)=0 \text { or } \operatorname{dim} H^{+}(B ; k)=0 .
$$

Now we establish (7.8) in case (iii). Recall that the Poincare series of a space $S$ is defined by

$$
f_{S}(t)=\sum_{p=0}^{\infty} \operatorname{dim} H^{p}(S ; k) t^{p} .
$$

Corollary 6.5 shows that

$$
f_{E}(t)=f_{B}(t) \cdot f_{F}(t)
$$

But (cf. [6, p. 492])

$$
f_{E}(t)=\left(1+t^{4}\right)\left(1+t^{2}+t^{4}\right) .
$$

Moreover, since $H^{*}(B ; k)$ and $H^{*}(F ; k)$ are evenly graded $f_{B}(t)$ and $f_{F}(t)$ are also polynomials in $t^{2}$. Now a simple check shows that were (7.8) to fail, then either

$$
f_{B}=1+t^{4} \text { and } f_{F}=1+t^{2}+t^{4}
$$

or

$$
f_{B}=1+t^{2}+t^{4} \text { and } f_{F}=1+t^{4} .
$$

By [7, Theorem 3] $H^{*}(B ; k)$ and $H^{*}(F ; k)$ are Poincaré duality algebras. Given the possibilities for the Poincare polynomials we can conclude that both algebras are truncated polynomial algebras in one variable. Hence the minimal models are given by

$$
\Lambda\left(b_{4}, b_{7}\right) ; d b_{7}=b_{4}^{2}, d b_{4}=0,
$$

and

$$
\Lambda\left(c_{2}, c_{5}\right) ; \quad d c_{5}=c_{2}^{3}, \quad d c_{2}=0 .
$$

(Subscripts denote degrees.)

Thus in this case the $\Lambda$-minimal $\Lambda$-model will have to provide a minimal model for $E$. But the minimal model for $E$ is (cf. [6, p. 475, Example 2]) $\left(\left(a_{2}\right.\right.$, $\left.a_{4}, a_{5}, a_{7}\right), D$ ) where

$$
D a_{2}=D a_{4}=0, \quad D a_{5}=a_{2}^{3}-2 a_{2} a_{4}, \quad D a_{7}=a_{4}^{2}-a_{2}^{2} a_{4} .
$$

Clearly $a_{2}^{3}$ is not a coboundary, and so there is no inclusion $\left(\Lambda\left(c_{2}, c_{5}\right), d\right)$ into $\left(\Lambda\left(a_{2}, a_{4}, a_{5}, a_{7}\right), D\right)$. An inclusion of $\left(\Lambda\left(b_{4}, b_{7}\right), d\right)$ into $\left(\Lambda\left(a_{2}, a_{4}, a_{5}, a_{7}\right)\right.$, $D$ ) would carry $b_{4}$ to $\alpha a_{2}^{2}+\beta a_{4}$ for some scalars $\alpha$ and $\beta$. But the space of coboundaries in degree 8 in $\left(\Lambda\left(a_{2}, a_{4}, a_{5}, a_{7}\right), D\right)$ is spanned by $a_{2}^{4}-2 a_{2}^{2} a_{4}$ 
and $a_{4}^{2}-a_{2}^{2} a_{4}$. It follows that $\left(\alpha a_{2}^{2}+\beta a_{4}\right)^{2}$ cannot be a coboundary (if $k \subset R)$.

Hence $\left(\Lambda\left(b_{4}, b_{7}\right), d\right)$ cannot be included in $\left(\Lambda\left(a_{2}, a_{4}, a_{5}, a_{7}\right), D\right)$ and so (7.9) and (7.10) are ruled out. This establishes (7.8) for (iii).

We now deduce the theorem from (7.8). Suppose first that $\operatorname{dim} H^{*}(B$; $k)=1$. Then Corollary 6.5 shows that

$$
j^{*}: H^{r}(E ; k) \rightarrow H^{r}(F ; k)
$$

is an isomorphism $(r=\operatorname{dim} E)$. Since $E$ is compact and orientable, $H^{r}(E$; $k) \neq 0$, but an element of $H^{r}(E ; k)$ will vanish on any proper subset. It follows that $F=E$ and $B=$ pt.

On the other hand, suppose $\operatorname{dim} H^{*}(F ; k)=1$. Then Corollary 6.5 shows that $H^{r}(B ; k) \neq 0$ and so $\operatorname{dim} B>r$.

Thus $\operatorname{dim} B=r=\operatorname{dim} E$. Hence (7.3) reads

$$
H^{p}(F, F-y ; k)= \begin{cases}0, & p>0 \\ k, & p=0\end{cases}
$$

Pick $y \in F$. Since $F$ is connected, $H^{0}(F ; k)=k$. Thus the exact sequence $0 \rightarrow H^{0}(F, F-y ; k) \rightarrow H^{0}(F ; k) \rightarrow H^{0}(F-y ; k) \rightarrow H^{1}(F, F-y ; k)$ shows that $H^{0}(F-y ; k)=0$. Hence $F-y=\varnothing, F=\{y\}$, and $E=B$. Q.E.D.

\section{REFERENCES}

1. C. Allday and S. Halperin, Lie group actions on spaces of finite rank, Quart. J. Math. Oxford Ser. (to appear).

2. A. Borel, Impossibilité de fibrer une sphère par un produit de sphères, C. R. Acad. Sci. Paris 231 (1950), 943-945.

3. H. Cartan, La transgression dans un groupe de Lie ..., Colloque de Topologie (espaces fibrés), Masson, Paris, 1951, pp. 57-71.

4. __ Theories cohomologiques, Invent. Math. 35 (1970), 261-272.

5. P. Grivel, Suite spectrale et modele minimal d'une fibration, Thèse, Université de Genève, 1977.

6. W. H. Greub et al., Connections, curoature and cohomology, Vol. III, Academic Press, New York, 1976.

7. S. Halperin, Finiteness in the minimal models of Sullivan, Trans. Amer. Math. Soc. 230 (1977), 173-199.

8. , Lectures on minimal models, Publ. Internes de L'U.E.R. de Math. Pures de l'Université de Lille I, no. 111, 1977.

9. J.-L. Koszul, Sur un type d'algèbres différentielles ..., Colloque de Topologie (espaces fibrés), Masson, Paris, 1951, pp. 73-81.

10. H. Shulman, Characteristic classes and foliations, Ph.D. Thesis, Univ. of California at Berkeley, 1972.

11. E. H. Spanier and J. H. C. Whitehead, On fibre spaces in which the fibre is contractible, Comment Math. Helv. 29 (1955), 1-7. 
12. D. Sullivan, Infinitesimal computations in topology, Inst. Hautes Etudes Sci. Publ. Math. 4 (1978), 269-331.

13. C. Watkiss, Cohomology of principal bundles in semisimplicial theory, Ph.D. Thesis, Univ. of Toronto, 1975.

Departagent of Phystcal sciences, Scarborovou College, Unteresty of Toronto, Toronto, Ontario MiC ia4 Canada 